\title{
Identification and modeling of a drug target for Clostridium perfringens SM101
}

\author{
Gagan Chhabra", Pramila Sharma", Avishek Anant, Sachin Deshmukh, Himani Kaushik, Keshav Gopal, Nutan Srivastava, Neeraj \\ Sharma, Lalit C. Garg*
}

Gene Regulation Laboratory, National Institute of Immunology, Aruna Asaf Ali Marg, New Delhi - 110067, India; "Equal contribution; Dr. Lalit C. Garg - E-mail : lalit@nii.res.in; Tel.: +91 11 26703652; Fax :+91 11 26742125; *Corresponding author

\begin{abstract}
:
In the present study, comparative genome analysis between Clostridium perfringens and the human genome was carried out to identify genes that are essential for the pathogen's survival, and non-homologous to the genes of human host, that can be used as potential drug targets. The study resulted in the identification of 426 such genes. The number of these potential drug targets thus identified is significantly lower than the genome's protein coding capacity (2558 protein coding genes). The 426 genes of C. perfringens were further analyzed for overall similarities with the essential genes of 14 different bacterial species present in Database of Essential Genes (DEG). Our results show that there are only 5 essential genes of $C$. perfringens that exhibit similarity with 12 species of the 14 different bacterial species present in DEG database. Of these, 1 gene was similar in 12 species and 4 genes were similar in 11 species. Thus, the study opens a new avenue for the development of potential drugs against the highly pathogenic bacterium. Further, by selecting these essential genes of $C$. perfringens, which are common and essential for other pathogenic microbial species, a broad spectrum anti-microbial drug can be developed. As a case study, we have built a homology model of one of the potential drug targets, ABC transporter-ATP binding protein, which can be employed for in silico docking studies by suitable inhibitors.
\end{abstract}

Received December 14, 2009; Revised December 26, 2009; Accepted December 28, 2009; Published January 17, 2010

Keywords: Clostridium perfringens, DEG, Essential genes, Drug targets, Broad-spectrum anti microbial drug.

\section{Background:}

The availability of the complete genome sequence information of the human genome and a large number of microbial genomes' sequences has led to the development of new approaches to understand hostpathogen interaction. Use of bioinformatics approach and comparative analysis of the genome of a pathogenic microbe allows one to identify essential genes necessary for the survival of that pathogen. The proteins encoded by these essential genes, that are not present or are nonhomologous to the host, can be used as drug targets. Such an approach has been effectively used to identify drug targets in other bacterial species such as Pseudomonas aeruginosa [1, 2], Helicobacter pylori [3], Mycobacterium tuberculosis [4], Burkholderia pseudomallei [5] and Aeromonas hydrophila [6]. Clostridium perfringens is a Grampositive, rod shaped, anaerobic bacterium that is able to form spores. It is widely distributed in the environment (e.g. in soil and sewage) and is frequently found in the gastrointestinal (GI) tract of humans, many domestic and feral animals, as well as in soil and freshwater sediments [7]. In humans, it can cause gangrene and gastrointestinal disease (e.g. food poisoning and necrotic enteritis), whereas in other animals, gastrointestinal and enterotoxemic diseases occur more frequently [8]. C. perfringens does not invade healthy cells but produces various toxins and enzymes that are responsible for associated lesions and symptoms. As a species, $C$. perfringens is one of the most prolific producers of toxins [9]. It has five biotypes (A, B, C, D and E), which are identified by the main types of toxins they produce (alpha, beta, iota, epsilon and theta), each type of toxin being associated with a specific syndrome. $C$. perfringens type $\mathrm{A}$ is the most common toxin type in the environment, and is responsible for gas gangrene, enterocolitis, dysenteria, and enterotoxemia. In the present study, comparative genome analysis of $C$. perfringens type $\mathrm{A}$ with that of the human genome, and use of the Database of Essential Genes (DEG) compiled by Zhang et al., [10], have resulted in the identification of the essential genes of $C$. perfringens, that could be used as potential drug targets.

Methodology:

Comparative genome analysis:

The complete genomes of C. perfringens type A, strain SM101 (Accession No. NC_008262) [11] and its human host have been sequenced and were downloaded from the NCBI website [12]. The Database of Essential Genes [10] was accessed from its website [13] and sequence alignment was performed using BLASTP. In this analysis, the assumption described by Dutta et al., [3] was followed, and proteins

ISSN 0973-2063 (online) 0973-8894 (print)

Bioinformation 4(7): 278-289 (2010) of less then 100 amino acid residues were excluded from the analysis. The remaining proteins were subjected to BLASTP on the NCBI server against human protein sequences to identify non-homologous sequences [14]. A minimum bit score of 100 and an Expectation value (E-value) cutoff of $10^{-10}$ was selected for shortlisting genes. The shortlisted genes were subjected to BLASTP against DEG to identify essential genes. Further analysis of the essential genes using the Kyoto Encyclopaedia of Genes and Genomes (KEGG) pathway database [15], revealed the information about different biological process in which potential target genes were involved.

\section{Structure modeling and visualization of model:}

BLASTP analysis was used to identify the most suitable template for homology modeling of Clostridium perfringens $\mathrm{ABC}$ transporter, ATP binding protein (CpABC) (Accession No. YP_698054). Subsequent to BLASTP analysis, the sequences of the structures of $\mathrm{ABC}$ transporters available in the PDB were used. The available structure of $\mathrm{ABC}$ transporter from Methanococcus jannaschii (Mj0796) in the Protein Database (PDB entry $1 \mathrm{f3o}$, resolution $=2.70$, $\mathrm{R}$ value $=0.204$ ) was used as a template. The target and the template sequences were aligned using ClustalW. MODELLER [16], an automated comparative protein modeling program, was used for homology modeling to generate the 3D structure of $\mathrm{CpABC}$. Further, the structural model generated was visualized using the Swiss PDB viewer software [17].

\section{Validation of the generated model:}

Different structure verification servers such as PROCHECK [18], WHAT_CHECK [19], VERIFY3D [20] and ProSA [21] were used to evaluate the 3D-model. These verification programs validate the predicted structure by checking various parameters. While PROCHECK, a structure verification program that relies on Ramachandran plot [22], determines the quality of the predicted structure by assessing various parameters such as lengths, angles and planarity of the peptide bonds, geometry of the hydrogen bonds, and side chain conformations of protein structures as a function of atomic resolution, WHAT_CHECK, a subset of protein verification tools from the WHATIF [23], program extensively evaluates the stereochemical parameters of the residues in the model [24]. The Verify3D determines the compatibility of an atomic model (3D) with its own amino acid sequence (1D) by assigning a structural class based on its location and environment (alpha, beta, loop, polar, nonpolar etc.) and comparing the results to valid structures [25]. 


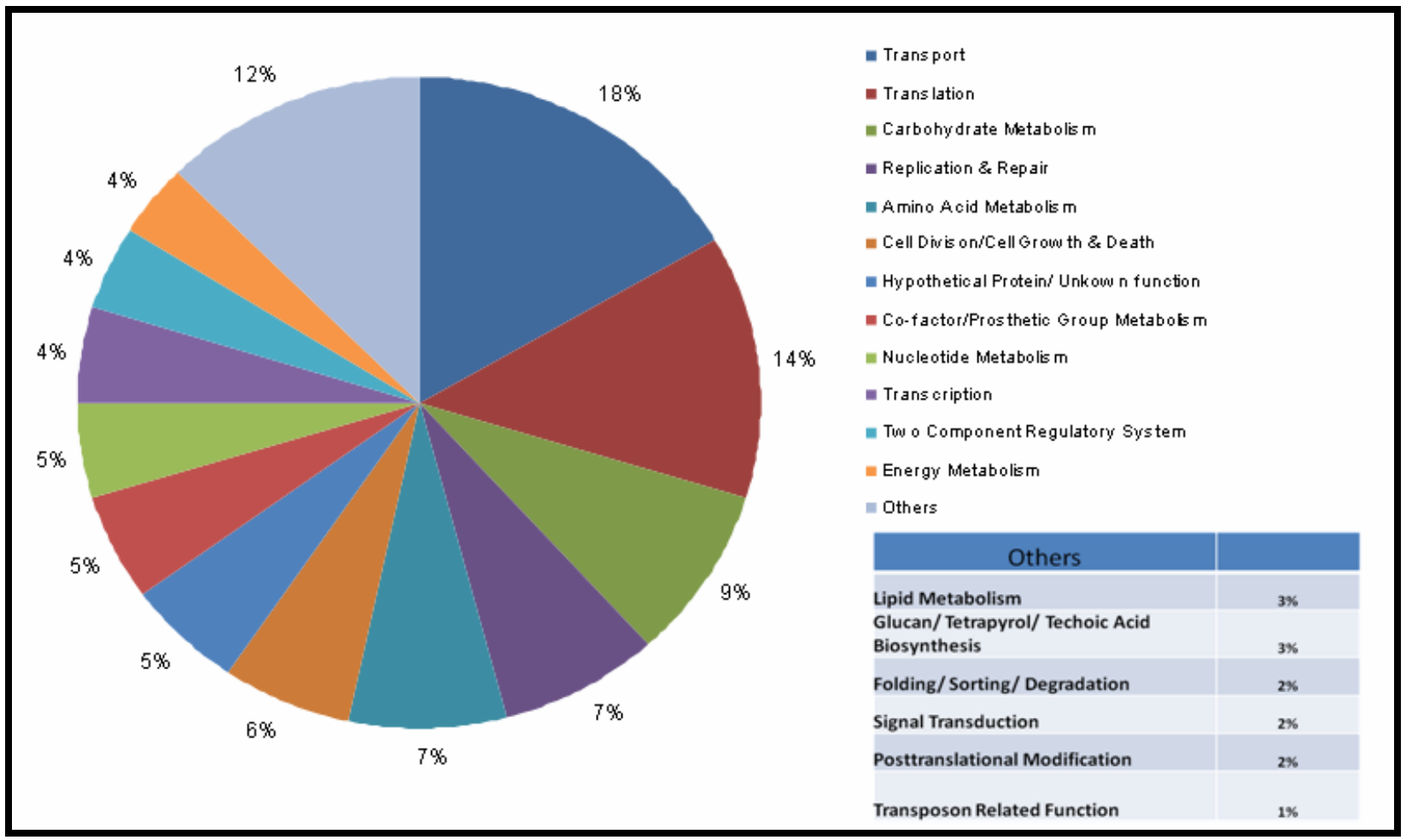

Figure 1: Percentage distribution of 426 target genes encoding different classes of proteins in Clostridium perfringens. Around $2 \%$ gene encode the proteins of unknown function.

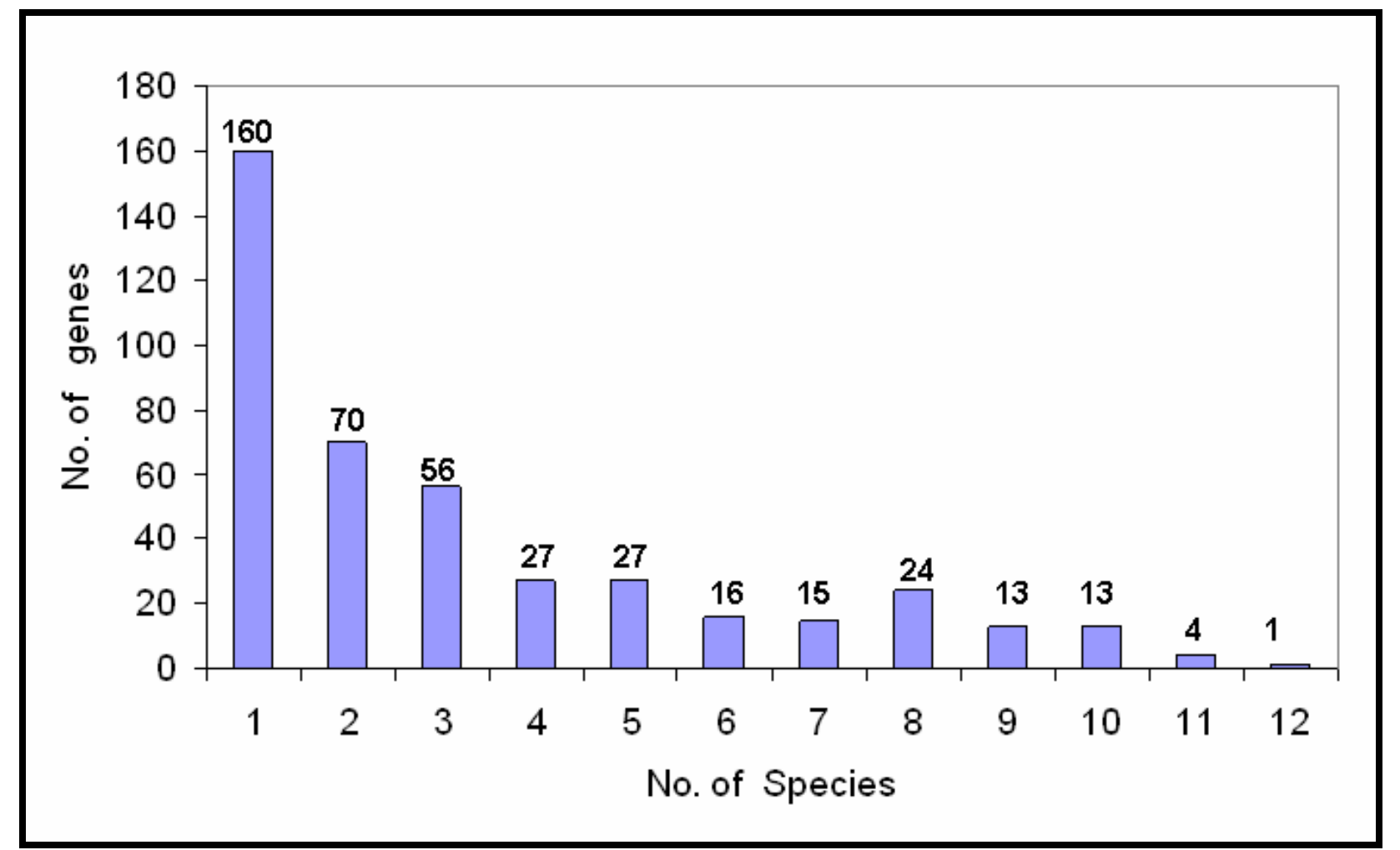

Figure 2: Graphical representation of the number of target genes aving similarity with those of bacterial species present in DEG. The number of genes of C. perfringens having similar match to different number of bacterial species is shown on top of the respective bars. 


$$
\begin{aligned}
& \text { Clostridium } \\
& \text { Mj0796 } \\
& \text { Clostridium } \\
& \text { 119Mj0796 } \\
& \text { Clostridium } \\
& \text { 171Mj0796 } \\
& \text { Clostridium } \\
& \text { Mj0796 }
\end{aligned}
$$

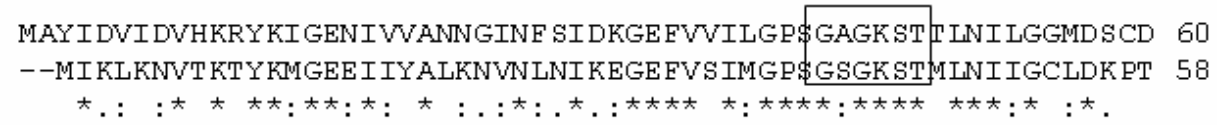

Figure 3: CLUSTALW Multiple sequence alignment of CpABC with Mj0796. Single fully conserved residues are represented by (*), conservation of strong and weak groups is denoted by (:) and (.), respectively. The boxed sequence represents the Walker A motif, whereas the ABC signature sequence is marked by bold overline.

\section{Discussion:}

\section{Identification of drug targets in $C$. perfringens:}

C. perfringens is the most common cause of gas gangrene in humans. Clostridial gas gangrene is a highly lethal necrotizing soft tissue infection of skeletal muscle caused by toxins secreted by $C$. perfringens. Although penicillin is one of the preferred antibiotics, it is only useful if the infection is diagnosed at early stages. There is no other specific drug that can be given to a patient infected with $C$. perfringens. Therefore, more research in this field is required to identify new drug targets and develop therapeutic agents for controlling $C$. perfringens infections. Since most antibiotics target essential cellular processes, essential gene products of microbial cells are promising new targets for antibacterial drugs [10]. Targetting an essential gene necessary for the bacterial cell survival may provide an effective way to control infection.

The circular genome of $C$. perfringens comprises 2,897,393 nucleotides with a total number of 2701 genes. Of the 2558 protein encoding genes, only 2300 genes encode proteins of greater than 100 amino acid residues. BLAST analysis of these genes against the human genome sequence revealed 1991 genes to be non-homologous to humans. Further BLASTP analysis of the 2300 protein coding genes with DEG resulted in identification of 726 genes, which had a bit score of at leas 100 at an expectation cutoff value of $10^{-10}$, as similar to the essential genes required for the growth and survival of bacteria listed in the DEG. Of these, 426 were found to have no human homologue (see Table 1 in Supplementary data). Pathways information for these genes was obtained from KEGG database. All these genes are involved in the production of proteins that are useful for various important functions in C. perfringens. Out of the 426 identified genes, function of 10 genes remains unknown, and 17 genes encode conserved hypothetical proteins. The percentage distribution of the genes amongst different biological process is shown in Figure 1. A large population of these genes $(\sim 33 \%)$ is involved in metabolic pathways. The major share of these genes constitute the proteins involved in transport and translation ( $17 \%$ and $12 \%$, respectively). Highly conserved genes, in theory, are more likely to be physiologically important [26]; however, they need to be experimentally validated. Therefore, the analysis of the 426 essential genes of C. perfringens for overall similarities with all 14 species present in DEG database was carried out. Results of such an analysis are shown in Figure 2. Out of the 426 essential genes, 160 genes have similar match to at least 1 species, whereas on the other end of the spectrum, only 4 genes have similar match to 11 species and only 1 gene has an identity score of more than 100 with 12 different microbial species listed in the DEG. From this analysis, it is evident that the products of 5 genes ( 1 gene similar in 12 species and 4 genes similar in 11 species) are essential for most of the bacterial species present in DEG. These species include Acinetobacter baylyi ADP1, Bacillus subtilis, Escherichia coli MG1655, Francisella novicida U112, Haemophilus influenzae, Helicobacter pylori 26695, Helicobacter pylori J99, Mycobacterium tuberculosis H37Rv, Mycoplasma genitalium G37, Mycoplasma pulmonis UAB CTIP , Salmonella typhimurium, Staphylococcus aureus and Streptococcus pneumoniae. Therefore, these 5 genes can be used as potential drug targets for more than 10 highly pathogenic bacterial species, in addition to $C$. perfringens. These 5 target genes, thus identified, are $\mathrm{ABC}$ transporterATP-binding protein, cell division protein FtsZ, RNA polymerase sigma factor RpoD, 50S ribosomal protein L13, and 30S ribosomal protein S5. A drug designed against these targets can be effectively used to treat other bacterial infections as well. Since the number of thus identified potential candidate genes is relatively small, these can be experimentally validated to develop broad-spectrum antimicrobial drugs. Since ABC transporter-ATP-binding protein is one of the 5 potential drug targets identified, an attempt has been made to predict its structure for effective drug design.

\section{ABC transporter - a potential broad spectrum target:}

ABC (ATP-binding cassette) transporters are ubiquitously present ATPdependent transmembrane solute pumps and ion channels. These superfamilies contain both uptake and efflux transport systems and form one of the largest transporters [27]. The ABC transporters couple hydrolysis of ATP to the translocation of various substrates across cell membranes. Members of this superfamily recognize substrates ranging from single ions to entire protein toxins. $\mathrm{ABC}$ transporters have a number of highly conserved $\mathrm{ABC}$ cassette motifs, many of which are involved in the binding and hydrolysis of ATP. It is generally assumed that all $\mathrm{ABC}$ cassettes bind and hydrolyze ATP in a similar way and use a common mechanism to provide energy for substrate transport through the membrane-spanning domains [28]. When the substrate has traversed the membrane, the transporter returns to the resting state through dissociation of ADP and inorganic phosphate. Fluoxetine and omeprazole, few of the most widely prescribed drugs in the world, have a transporter protein as site of action. Therefore, $\mathrm{ABC}$ transporter structures have potential value in drug designing. 
A

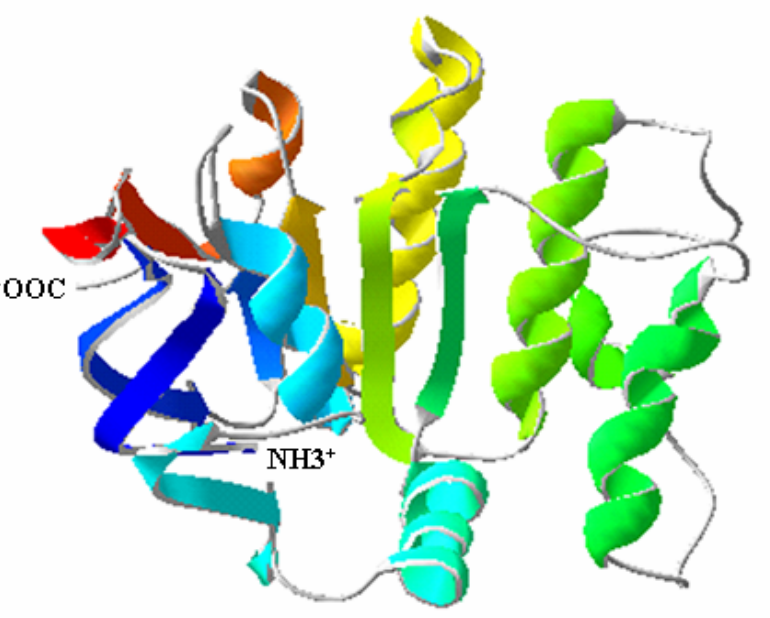

B

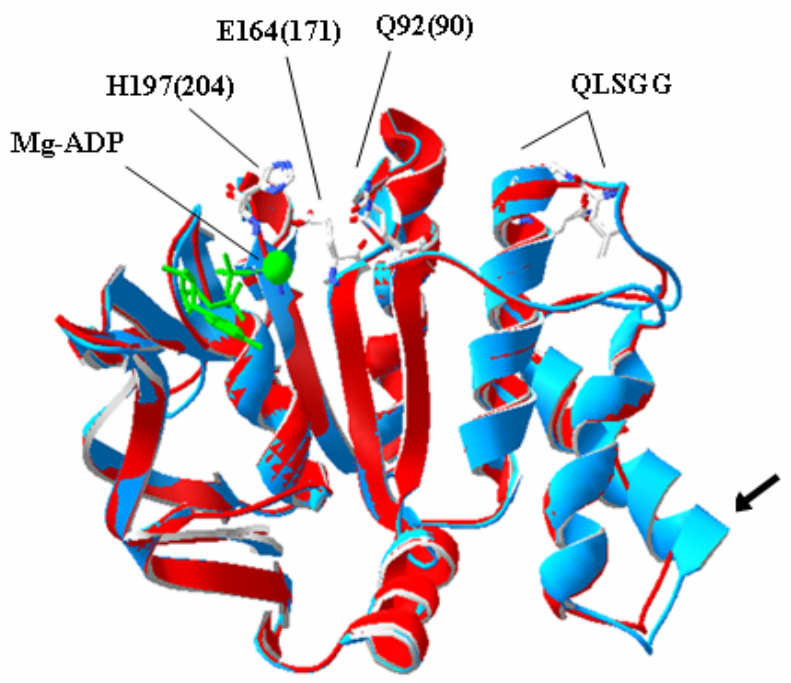

Figure 4: (A): Homology modeled structure of the C. perfringens ABC transporter, ATP binding protein based on template Mj0796. Model is represented in ribbon form as Swiss PDB viewer representation in secondary structure succession color scheme. N and C termini of modeled structure are represented as $\mathrm{NH}_{3}{ }^{+}$and ${ }^{-}$OOC. (B) Superimposed image of the modeled structure of CpABC onto Mg-ADP bound Mj0796 (PDB entry $1 \mathrm{f3o}$ ). CpABC is represented in red color, Mg-ADP (shown in green color) bound Mj0796 is represented in blue color. Signature sequence LSGG with three conserved residues, Q90, E171, H204 form Mj0796 and Q92, E164, H197 from model CpABC are mentioned. Arrow indicates the deletion of seven amino acids (RKRALEC) in CpABC, which forms an $\alpha$-helix in the Mj0796.

Structural model and overall architecture of $\mathrm{ABC}$ transporter (СрABC):

Sequence alignment of the $C$. perfringens $\mathrm{CpABC}$ and $\mathrm{ABC}$ transporters from other species revealed $\mathrm{Mj} 0796$ to be the best template for homology modeling of the target sequence as the $\mathrm{CpABC}$ and Mj0796 shared 41\% identity (Figure 3). Mj0796 is a member of the o228/LolD transporter family, involved in the export of lipoproteins via the Lol system. LolD contains a characteristic sequence called the LolD motif, which is highly conserved among LolD homologs, but not in other $\mathrm{ABC}$ transporters, and is located between the Walker A (GSGKST, boxed) and ABC signature (LSGGQ, marked as bold overline) motifs (Figure 3). Comparative sequence analyses, motif search, and secondary structure prediction indicated that $\mathrm{CpABC}$ is structurally similar to the monomer structure of the $\mathrm{Mj} 0796$, a lipid transporter. The crystal structure of Mj0796 (PDB entry 1f3o) was used as a template to predict the structure of $\mathrm{CpABC}$ and the predicted $3 \mathrm{D}$ structure model of $\mathrm{CpABC}$ was generated by Modeller, a homologymodeling program. Figure 4 shows the predicted structure in the form of ribbons as a Swiss PDB viewer representation.

Validation of generated model of CpABC:

The quality of the model was evaluated using the PROCHECK program and assessed using the Ramachandran plot. It is evident from the Ramchandran plot that the predicted model has $91.4 \%, 8.1 \%$, and $0.5 \%$ residues in the most favorable regions, the allowed regions, and the disallowed regions, respectively. Such a percentage distribution of the protein residues determined by Ramachandran plot shows that the predicted model is of good quality. All Ramachandrans show 6 labelled residues out of 220 whereas chi1-chi2 plots show 2 labelled residues out of 140. The model shows all the main chain and side chain parameters to be in the 'better' region. Another factor that is important for the predicted model to be reliable is G-factor, which is a log odds score based on the observed distribution of stereochemical parameters. For a reliable model, the score for G-factor should be above -0.50 . The observed G-factor score for the present model was -0.05 for dihedrals bonds, -0.31 for covalent bonds, and -0.15 overall. The distribution of the main chain bond lengths and bond angles were $98.5 \%$ and $93.2 \%$ within limits, respectively. Also, all the planar groups were within the limits. The quality of the generated model of $\mathrm{CpABC}$ as evaluated by ProSA provided a z-score of -7.2, which falls within the range of values observed for the experimentally determined structures of similar lengths. The validity of the predicted model of $\mathrm{CpABC}$ was also verified by employing the structure verification servers WHAT_CHECK and Verify-3D. Superposition of the predicted structure of CpABC and the Mg-ADP bound Mj0796 (template, PDB entry 1f3o) is shown in Figure 4B. It is evident from the figure that the $\mathrm{Mg}$-ADP binding core of the $\mathrm{ABC}$ subunit and all the structural motifs are highly conserved in both structures. The two superposed structures match $214 \mathrm{C} \alpha$ atoms with an rms distance of $0.47 \AA$. Three residues Q90, E171, and H204, important for activity of Mj0796, superposed very well with conserved residues Q92, E164 and H197 from model CpABC. However, a deletion of seven amino acids (RKRALEC), which forms an $\alpha$-helix in the Mj0796 (indicated by arrow), and an insertion of three amino acids (PIS) at the C-terminal end of $\mathrm{CpABC}$, is observed. Thus, the predicted model structure of $C$. perfringens $\mathrm{ABC}$ transporter, ATP binding protein, and $\mathrm{CpABC}$ is comparable to the structurally resolved Mj0796.

\section{Conclusion:}

Comparative genome analysis is a highly efficient approach for idendifing potential proteins that can be used as potential targets for effective drug designing against pathogenic organisms. It allows restricting the potential pool of genes to a much smaller number, compared to the whole genome capacity, which can be experimentally validated. In the present study, around 426 drug targets in $C$. perfringens were identified by comparative genome analysis with DEG. 
Further, by using the subtractive genomic approach five essential genes were identified that are conserved in more than 10 other pathogenic organisms. Since the number of these conserved genes is very small, these can be experimentally tested for the development of a broadspectrum anti-microbial drug. The drug thus developed is likely to inhibit other bacterial infections, which share high sequence similarity with the five essential genes of $C$. perfringens SM101.

\section{Acknowledgements:}

Financial assistance from the Department of Biotechnology, New Delhi, India is acknowledged. The Council of Scientific and Industrial Research, New Delhi, India, and the University Grants Commission, New Delhi, India is gratefully acknowledged for senior research fellowships to AA and $\mathrm{KG}$, respectively.

\section{References:}

[1] KR Sakharkar et al., In Silico Biology 4:355 (2004) [PMID: 15724285]

[2] D Perumal et al., In Silico Biology 7:453 (2007) [PMID: 18391237]

[3] A Dutta et al., In Silico Biology 6:43 (2006) [PMID: 16789912]

[4] S Anishetty et al., Computational Biology and Chemistry 29:368 (2005) [PMID: 16213791]

[5] CE Chong et al., In Silico Biology 6:341(2006) [PMID: 16922696]

[6] V Sharma et al., In Silico Biology 8:331 (2008) [PMID: 19032165]

[7] JI Rood, ST Cole, Microbiology Review 55:621 (1991) [PMID: 1779929]

[8] JG Songer, Clin. Microbiology Review 9:216-234 (1996) [PMID: 8964036]
[9] JI Rood, Annual Review of Microbiology 52:333 (1998) [PMID: 9891801]

[10] R Zhang et al., Nucleic Acids Research 32:D271 (2004) [PMID:14681410]

[11] GS Myers et al., Genome Research 16:1031 (2006) [PMID: 16825665]

[12] http://www.ncbi.nlm.nih.gov/

[13] http://tubic.tju.edu.cn/deg/

[14] SF Altschul et al., Journal of Molecular Biology 215:403 (1990) [PMID: 2231712]

[15] H Ogata et al., Nucleic Acids Research 27:29 (1999) [PMID: 9847135]

[16] A Fiser, A Sali, Methods in Enzymology 374:461 (2003) [PMID: 14696385]

[17] http://www.expasy.org/spdbv

[18] RA Laskowski et al., Journal of Applied Crystallography 26:283 (1993)

[19] RWW Hooft et al., Nature 381:272 (1996) [PMID: 8692262]

[20] D Eisenberg et al., Enzymology 277:396 (1997) [PMID: 9379925]

[21] M Wiederstein, MJ Sippl, Nucleic Acids Research, 35 (Web Server issue), W407 (2007) [PMID: 17517781]

[22] GN Ramachandran, V Sasisekharan, Advances in Protein Chemistry 23:283 (1968) [PMID: 4882249]

[23] G Vriend, Journal of Molecular Graphics 8:52 (1990) [PMID: 2268628]

[24] http://swift.cmbi.ru.nl/gv/whatcheck/

[25] http://nihserver.mbi.ucla.edu/Verify_3D/

[26] F Arigoni, Nature Biotechnology 16:851 (1998) [PMID: 9743119]

[27] MH Saier, Jr, Microbiology 146:1775 (2000) [PMID: 10931885]

[28] KP Locher et al., Science 296:1091 (2002) [PMID: 12004122]

Edited by P. Kangueane

Citation: Chhabra et al., Bioinformation 4(7): 278-289 (2010)

License statement: This is an open-access article, which permits unrestricted use, distribution, and reproduction in any medium, for noncommercial purposes, provided the original author and source are credited.
ISSN 0973-2063 (online) 0973-8894 (print) Bioinformation 4(7): 278-289 (2010) 


\section{Supplementary material}

Table 1: List of genes that are non-homologs to humans and essential for $C$. perfringens

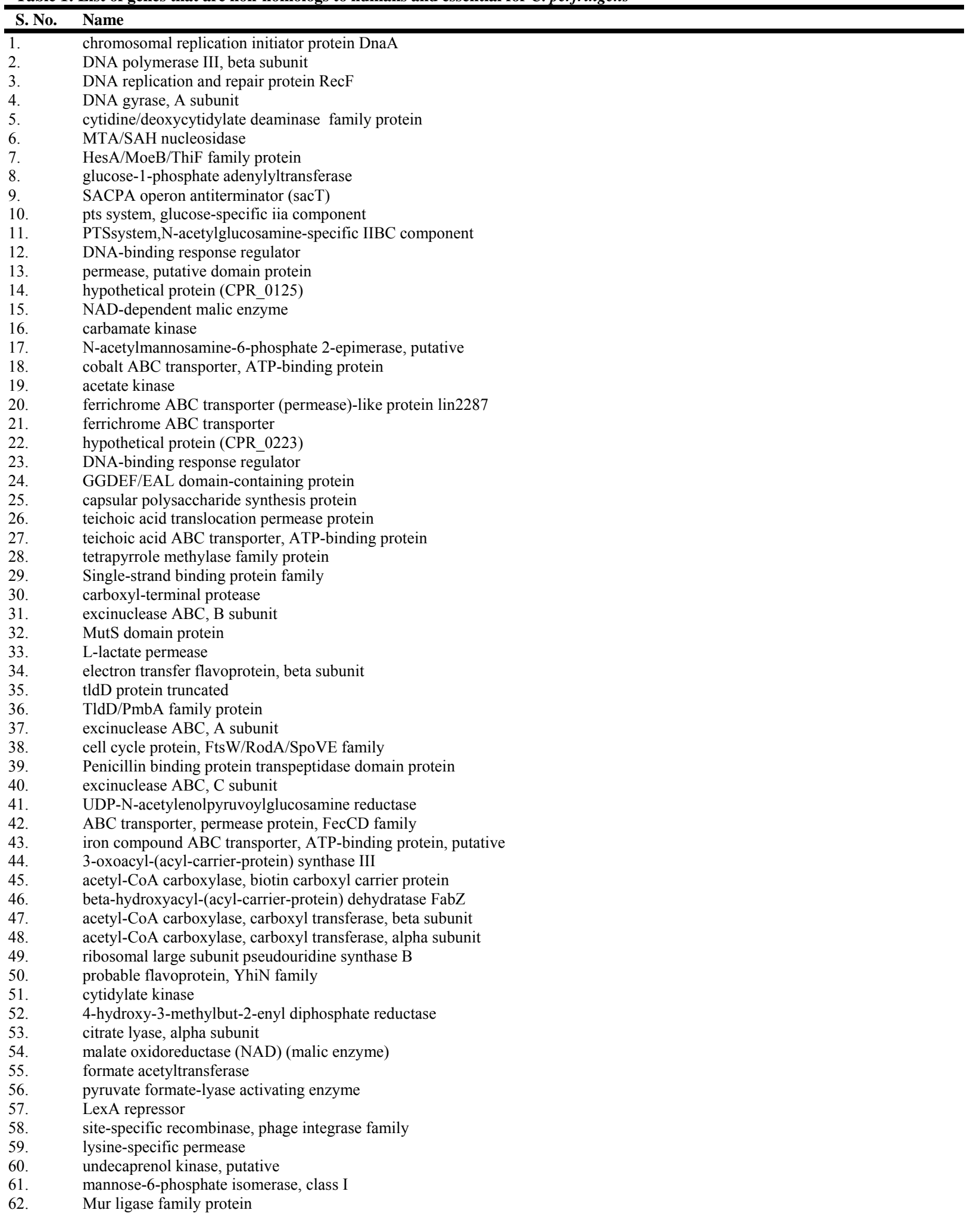


63. CobB/CobQ family glutamine amidotransferase

64. stage $\mathrm{V}$ sporulation protein $\mathrm{B}$

65. pseudouridylate synthase

66. amidohydrolase family protein

67. ATP-dependent DNA helicase PcrA, putative

68. uracil permease

69. drug resistance transporter, EmrB/QacA family

70. magnesium transporter

71. NADPH-dependent butanol dehydrogenase

72. oligopeptide transporter, OPT family

73. hypothetical protein (CPR_1285)

74. SsrA-binding protein

75. phosphoglycerate mutase, 2,3-bisphosphoglycerate-independent

76. RNA polymerase sigma-54 factor

77. primosome assembly protein PriA

78. conserved hypothetical protein (CPR_1721)

79. L-asparaginase, type II

80. sensory box histidine kinase

81. transcriptional regulator, NrdR family

82. RNA polymerase sigma-G factor

83. cell division protein FtsZ

84. cell division protein FtsA

85. twitching motility protein PilT

86. peptidase, U32 family

87. conserved hypothetical protein (CPR_1743)

88. RNA-metabolizing metallo-beta-lactamase family protein

89. segregation and condensation protein $\mathrm{B}$

90. segregation and condensation protein A

91. tyrosine recombinase XerD

92. $\quad \mathrm{NAD}(+) / \mathrm{NADH}$ kinase

93. hemolysin A

94. geranyltranstransferase

95. translation elongation factor $\mathrm{P}$

96. pilus biogenesis protein, putative

97. diaminopimelate epimerase

98. methyltransferase, putative

99. stage $\mathrm{V}$ sporulation protein $\mathrm{E}$

100. phospho-N-acetylmuramoyl-pentapeptide-transferase

101. UDP-N-acetylmuramoyl-tripeptide--D-alanyl-D-alanine ligase

102. udp-n-acetylmuramoylalanyl-d-glutamate--2,6-diaminopimelate

103. stage V sporulation protein D, spoVD, FtsI/pbp family

104. teichoic acid biosynthesis protein $\mathrm{A}$

105. stage V sporulation protein D, spoVD, FtsI/pbp family

106. MutS2 family protein

107. potassium uptake protein, TrkH family

108. ribosomal protein L20

109. translation initiation factor IF-3

110. aspartate-semialdehyde dehydrogenase

111. dihydrodipicolinate reductase

112. cob(I)alamin adenosyltransferase, putative

113. 2,3,4,5-tetrahydropyridine-2,6-dicarboxylate $\mathrm{N}$-succinyltransferase, putative

114. membrane proteins-like protein lmo0908, putative

115. bacterial extracellular solute-binding proteins, family 5 superfamily

116. bacterial type II secretion system protein $\mathrm{F}$

117. secretion system protein $\mathrm{E}$

118. hypothetical protein (CPR_2297)

119. ribosomal large subunit pseudouridine synthase $\mathrm{f}$

120. 2-C-methyl-D-erythritol 2,4-cyclodiphosphate synthase

121. phosphoglucosamine mutase

122. sensor protein yycg

123. two-component response regulator

124. 4-alpha-glucanotransferase

125. transcriptional regulator, LacI family

126. conserved hypothetical protein (CPR_2346)

127. thioredoxin reductase

128. phosphoenolpyruvate-protein phosphotransferase

129. ribosomal protein S4

130. ribonucleotide-diphosphate reductase subunit beta 
sensor histidine kinase

DNA-binding response regulator

ribosomal protein $\mathrm{S} 9$

ribosomal protein L13

$\mathrm{ABC}$ transporter permease protein

ribosomal protein L17

DNA-directed RNA polymerase, alpha subunit

ribosomal protein $\mathrm{S} 4$

ribosomal protein $\mathrm{S} 11$

ribosomal protein $\mathrm{S} 13 \mathrm{p} / \mathrm{S} 18 \mathrm{e}$

preprotein translocase, SecY subunit

ribosomal protein L15

ATP-binding protein

hypothetical protein (CPR 0337)

DNA polymerase III, alpha subunit, interruption-C

cardiolipin synthetase

phosphopentomutase

quinolinate synthetase complex, A subunit

PTS system, IIB component

LacI family transcription regulator

PTS system, IIBC component

helicase/exonuclease

tRNA (guanine-N(7)-)-methyltransferase

$\mathrm{ABC}$ transporter domain protein

DNA-binding response regulator

glycosyltransferase, putative

beta-1,4-N-acetyl-mannosaminyltransferase, putative

polysaccharide transporter protein, putative

UTP-glucose-1-phosphate uridylyltransferase

UTP-glucose-1-phosphate uridylyltransferase

$\mathrm{ABC}$ transporter, permease protein

hypothetical protein (CPR_0491)

NAD-dependent 4-hydroxybutyrate dehydrogenase

permease

nitrite/sulfite reductase-like protein

stage V sporulation protein D

riboflavin biosynthesis protein RibD

riboflavin synthase, alpha subunit

riboflavin biosynthesis protein RibA

phosphate permease

fructose specific permease

1-phosphofructokinase

diaminopimelate decarboxylase

amino acid $\mathrm{ABC}$ transporter, permease protein-like protein lin2352

cation efflux family protein

transporter-like protein lin 1189

glycosyltransferase

polysaccharide biosynthesis protein, putative

DNA-binding response regulator

glutaminyl-tRNA synthetase

tyrosyl-tRNA synthetase

relA/spoT family protein

BirA bifunctional protein

degV family protein

rRNA methylase-like protein cspR

autolytic lysozyme, putative

sensory box histidine kinase

endonuclease III

serine $\mathrm{O}$-acetyltransferase

amino acid (glutamine) ABC transporter, permease protein-like protein lin 1851

amino acid ABC transporter ATP-binding protein

putative lipid kinase

phosphomethylpyrimidine kinase

transcriptional regulator, LacI family

d-galactose-binding periplasmic protein precursor

Galactoside transport ATP-binding protein mglA

beta-methylgalactoside transporter inner membrane component

fructose-1,6-bisphosphate aldolase, class II 
aminotransferase family protein

200. GTPase EngB

201. purine nucleoside phosphorylase

202. thiamine biosynthesis/tRNA modification protein ThiI

203.

204.

205.

206 .

207.

sodium/alanine symporter

cardiolipin synthase

glutamyl-tRNA reductase

oligoendopeptidase $\mathrm{F}$

aminotransferase, class $\mathrm{V}$

deoxyuridine 5'-triphosphate nucleotidohydrolase

sulfate permease, SulP family

nitrate $\mathrm{ABC}$ transporter ATP binding protein

uracil transporter

sucrose-6-phosphate hydrolase

sucrose operon repressor

spoVK domain protein

molybdopterin oxidoreductase

undecaprenyl diphosphate synthase, putative

ABC transporter (permease proteins)-like protein $1 \mathrm{mo} 1390$

glycogen branching enzyme

DNA-binding response regulator

sensor histidine kinase

GTP pyrophosphokinase

protein-export membrane protein $\mathrm{SecF}$

protein-export membrane protein $\mathrm{SecD}$

holliday junction DNA helicase RuvB

holliday junction DNA helicase RuvA

conserved hypothetical protein (CPR_1923)

hypoxanthine phosphoribosyltransferase

membrane carboxypeptidase $\mathrm{mrcB}$

sodium/alanine symporter family protein

spermidine/putrescine $\mathrm{ABC}$ transporter, permease protein (potB)-like protein

spermidine/putrescine $\mathrm{ABC}$ transporter, permease protein (potC)-like protein

spermidine/putrescine-binding periplasmic protein precursor

aspartate--ammonia ligase

lrgB-like family protein

aminopeptidase

hypothetical protein (CPR_1972)

hypothetical protein (CPR_1973)

RNA polymerase sigma factor RpoD

DNA primase

pyruvate,phosphate dikinase

GTP-binding protein Era

hypothetical protein (CPR_1998)

ribosomal protein L11 methyltransferase

heat-inducible transcription repressor HrcA

DNA polymerase III, delta subunit

ATP-dependent protease

deoxyribose-phosphate aldolase

manganese-dependent inorganic pyrophosphatase, putative

UDP-N-acetylglucosamine- -N-acetylmuramyl-(pentapeptide) pyrophosphoryl-undecaprenol N-acetylglucosamine transferase

DNA topoisomerase, GyrA/ParC subunit family

ROK family protein

sugar transport system (permease) (binding protein dependent transporter)

FemAB family protein

glutamine-binding periplasmic protein of glutamine $\mathrm{ABC}$ transporter

vncR, response regulator

ABC transporter (ATP binding protein)-like protein 1 mo1063

ribosomal protein $\mathrm{S} 5$

ribosomal protein L18

ribosomal protein L6

ribosomal protein $\mathrm{S} 8$

ribosomal protein L5

ribosomal protein L14

$50 \mathrm{~S}$ ribosomal protein L16

30S ribosomal protein $\mathrm{S} 3$

ribosomal protein L22

ribosomal protein L3 
ribosomal protein $\mathrm{S} 10$

ribosomal protein $\mathrm{S} 7$

ribosomal protein $\mathrm{S} 12$

ribosomal protein $\mathrm{L} 7 / \mathrm{L} 12$

50 S ribosomal protein L10

ribosomal protein $\mathrm{L} 1$

ribosomal protein L11

transcription termination/antitermination factor NusG

thymidylate synthase, flavin-dependent

2-C-methyl-D-erythritol 4-phosphate cytidylyltransferase

DNA repair protein RadA

transcriptional regulator, GntR family

thioredoxin-disulfide reductase

UDP-N-acetylmuramoylalanine--D-glutamate ligase

transcription elongation factor GreA

tRNA(Ile)-lysidine synthetase

MazG family protein

stage $\mathrm{V}$ sporulation protein $\mathrm{B}$

thiamine biosynthesis protein $\mathrm{ThiC}$

phosphoribosylaminoimidazole carboxylase, catalytic subunit

3-dehydroquinate synthase

chorismate synthase

3-dehydroquinate dehydratase, type II

hypothetical protein (CPR_0697)

probable permease, putative

Cytochrome $\mathrm{C}$ biogenesis protein transmembrane region family

$\mathrm{ABC}$ transporter, ATP-binding protein

cardiolipin synthetase

sodium:glactoside symporter family protein

antibiotic ABC transporter ATP binding protein SSO1934

acyl carrier protein phosphodiesterase

iron compound $\mathrm{ABC}$ transporter, permease protein

iron(III) dicitrate transport permease-like protein yusV

cymH protein

iron compound $\mathrm{ABC}$ transporter, permease protein

ferrichrome transport system, permease protein

Ferrichrome transport ATP-binding protein fhuC

ISCpe6, transposase orfA

NADH-dependent butanol dehydrogenase a

GntP family permease

uncharacterized conserved protein, YHAD family

MATE efflux family protein

thermophilic metalloprotease family protein

sucrose-6-phosphate hydrolase e 1

PTS system, N-acetylglucosamine-specific IIBC component

cation efflux family protein

DNA-binding response regulator

pyridine nucleotide-disulphide oxidoreductase family protein

triple helix repeat-containing collagen

sensory box histidine kinase/response regulator

L-serine dehydratase, iron-sulfur-dependent, alpha subunit

oxidoreductase, 2-nitropropane dioxygenase family

$\mathrm{HPr}(\mathrm{Ser})$ kinase/phosphatase

methylglyoxal synthase

Predicted metal-dependent phosphoesterase (PHP family)

para-aminobenzoate synthase glutamine amidotransferase, component II

para-aminobenzoate synthase, component I

dihydropteroate synthase

dihydroneopterin aldolase/ 2-amino-4-hydroxy-6-hydroxymethyldihydropteridine pyrophosphokinase

thiazole biosynthesis protein ThiG

transporter, major facilitator family

methionine-R-sulfoxide reductase

transcriptional regulator, LacI family

teichoic acid linkage unit synthesis protein-like protein lin2663

ferripyochelin binding protein

HD superfamily hydrolase, YMDA

recA protein

CDP-diacylglycerol--glycerol-3-phosphate 3-phosphatidyltransferase 
335.

336.

337.

338.

339.

340.

341.

342.

343.

344.

345 .

346.

347.

348 .

349.

350

351 .

352

353.

354

355.

356.

357.

358 .

359.

360 .

361 .

362.

363.

364.

365

366.

367.

368 .

369

370 .

371 .

372.

373

374.

375 .

376.

377.

378.

379.

380.

381 .

382

383.

384.

385.

386

387.

388 .

389 .

390

391.

392.

393.

394.

395.

396.

397.

398.

399

400.

401

402.
DNA translocase FtsK

aspartokinase

riboflavin biosynthesis protein RibF

DHH subfamily 1 protein

DNA polymerase III, alpha subunit, Gram-positive type

4-hydroxy-3-methylbut-2-en-1-yl diphosphate synthase

1-deoxy-D-xylulose 5-phosphate reductoisomerase

phosphatidate cytidylyltransferase

ribosome recycling factor

uridylate kinase

translation elongation factor Ts

ribosomal protein $\mathrm{S} 2$

GTP-sensing transcriptional pleiotropic repressor CodY

DNA protecting protein DprA

ribonuclease HII

ribosomal protein L19

radical SAM domain-containing protein

fatty acid/phospholipid synthesis protein PlsX

acetate kinase

phosphate acetyltransferase

pantetheine-phosphate adenylyltransferase

ATP-dependent DNA helicase RecG

DAK2 domain protein

GTPase YjeQ

protein phosphatase 2C family protein

polypeptide deformylase

pseudouridylate synthase family protein, yabo

nicotinate nucleotide adenylyltransferase

ribosomal protein L27

ribonuclease, Rne/Rng family

cell cycle protein, FtsW/RodA/SpoVE family

septum site-determining protein MinD

Penicillin-binding Protein dimerisation domain family

rod shape-determining protein $\mathrm{MreB}$

DNA repair protein, RadC family

hypothetical protein (CPR_2114)

aminoacyl-histidine dipeptidase

hypothetical protein (CPR_2119)

PTS system, glucose-specific IIBC component

$\mathrm{ABC}$ transporter, substrate-binding protein

conserved hypothetical protein (CPR_2132)

glycoprotease family protein

preprotein translocase, SecA subunit

RecD/TraA family helicase

$\mathrm{mbl}$ protein

UDP-N-acetylglucosamine 1-carboxyvinyltransferase

UDP-N-acetylglucosamine 2-epimerase

uracil phosphoribosyltransferase

ribose 5-phosphate isomerase b

$\mathrm{Sua} / \mathrm{YciO} / \mathrm{YrdC} / \mathrm{YwlC}$ family protein

modification methylase, HemK family

thymidine kinase

transcription termination factor Rho

4-diphosphocytidyl-2C-methyl-D-erythritol kinase

sensor histidine kinase HpkA

DNA-binding response regulator

aminoacyl-histidine dipeptidase

non-canonical purine NTP pyrophosphatase, rdgB/HAM1 family

ribonuclease $\mathrm{PH}$

glucose-6-phosphate isomerase

oligopeptide ABC transporter, ATPase component

oligopeptide ABC transporter, ATPase component

oligopeptide $\mathrm{ABC}$ transporter, permease component

oligopeptide $\mathrm{ABC}$ transporter, permease component

transcription-repair coupling factor

PDZ domain protein

sensor histidine kinase

DNA-binding response regulator

ISSN 0973-2063 (online) 0973-8894 (print)

Bioinformation 4(7): 278-289 (2010) 


\section{Bioinformation}

403. UDP-N-acetylglucosamine pyrophosphorylase

404. UDP-N-acetylmuramate--alanine ligase

405. Transcriptional regulator

406. metallopeptidase, family M24

407. primase-like protein

408. bifunctional acetaldehyde-CoA/alcohol dehydrogenase

409. catabolite control protein A, putative

410. glycerol uptake operon antiterminator

411. glutamate racemase

412. glutamine synthetase, putative

413. Transcriptional regulator

414. metallo-beta-lactamase family protein

415. UDP-N-acetylglucosamine 1-carboxyvinyltransferase

416. YhiN family flavoprotein

417. L-serine dehydratase, iron-sulfur-dependent, alpha subunit

418. class II aldolase, tagatose bisphosphate family

419. replicative DNA helicase

420. ATP-dependent protease

421. ribosomal protein L9

422. DHH family protein

423. stage 0 sporulation protein $\mathrm{J}$

424. sporulation initiation inhibitor protein soj

425. methyltransferase GidB

426. membrane protein OxaA 